EPJ Web of Conferences 41, 04003 (2013)

DOI: $10.1051 /$ epjconf/20134104003

(C) Owned by the authors, published by EDP Sciences, 2013

\title{
Observation of exciton-polariton ultrafast dynamic Stark effect
}

Christoph Lange ${ }^{1}$, Alex Hayat ${ }^{1}$, Lee A. Rozema ${ }^{1}$, Ardavan Darabi ${ }^{1}$, Henry M. van Driel ${ }^{1}$, Aephraim M. Steinberg ${ }^{1}$, Bryan Nelsen ${ }^{2}$, David W. Snoke ${ }^{2}$, Loren N. Pfeiffer ${ }^{3}$ and Kenneth W. West ${ }^{3}$

${ }^{1}$ Department of Physics, Centre for Quantum Information and Quantum Control, and Institute for Optical Sciences, University of Toronto, Toronto, Ontario M5S 1A7, Canada

${ }^{2}$ Department of Physics and Astronomy, Univ. of Pittsburgh, Pittsburgh, Pennsylvania 15260, USA

${ }^{3}$ Department of Electrical Engineering, Princeton University, Princeton, New Jersey 08544, USA

\begin{abstract}
We demonstrate ultrafast phase control of exciton-polaritons in a $\mathrm{GaAs} / \mathrm{AlGaAs}$ strongly coupled microcavity exploiting the ac Stark effect. Our approach yields $\mathrm{meV}$-scale shifts without carrier generation, providing a powerful tool towards control of polariton BECs.
\end{abstract}

Strong light-matter coupling has become a rapidly growing field ever since the first demonstration of vacuum Rabi splitting [1,2] in cold atom systems. In semiconductor microcavities, quantum well (QW) excitons are strongly coupled to a cavity mode [3] and exciton-polaritons arise. Their extremely low effective mass yet strong mutual interactions have enabled the observation of a multitude of physics such as high-temperature Bose-Einstein condensation (BEC) $[4,5]$.

The prospect of controlling properties of these condensates such as the local phase has stimulated several experiments recently, exploiting repulsive exciton interactions to create long-range order [6] or to generate quantum-fluid vortices [7]. Relying on the generation of additional carriers, however,

(a)

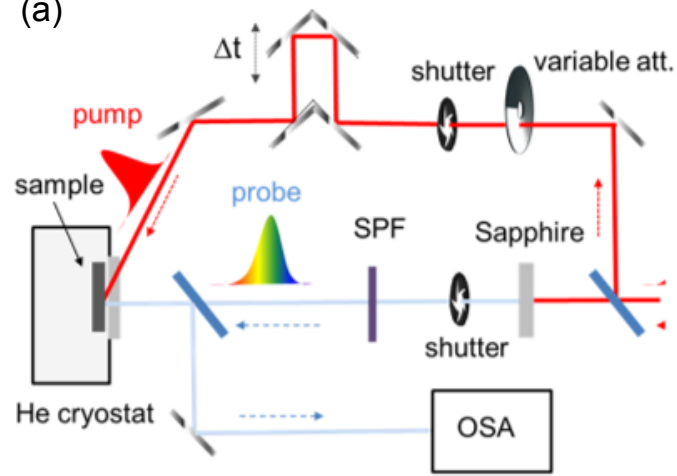

(b)

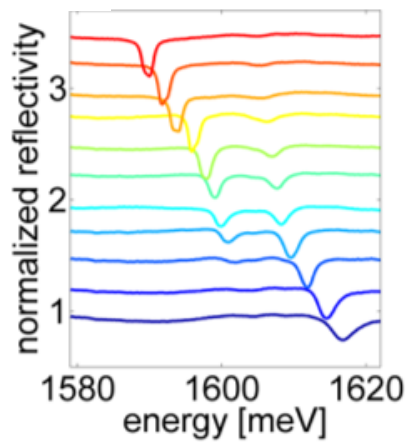

Fig. 1. Experimental pump-probe setup for measuring the dynamic Stark effect (a) and normalized reflectivity (b) along the taper direction, vertically offset for clarity. The single dip at the lowest and topmost spectrum moves through spectrally with the location on the sample, and anti-crossing with the exciton resonance is observed around $1.604 \mathrm{eV}$.

the switching time of exciton density potentials is directly bound to the carrier recombination time. Furthermore, they allow only limited spatial control due to diffusion and complicate the extraction of properties of the condensed particles alone.

Here, we exploit the ultrafast, non-resonant dynamical Stark shift to control the phase of exciton polaritons without carrier generation. Our non-invasive approach enables imprinting of phases on the polariton doublet whose energy structure remains fully intact, in line with similar experiments in cold-atom BEC [8].

This is an Open Access article distributed under the terms of the Creative Commons Attribution License 2.0, which permits unrestricted use, distribution, and reproduction in any medium, provided the original work is properly cited. 
We use a $250 \mathrm{kHz}$ amplifier which delivers $200 \mathrm{fs}$ pulses at $800 \mathrm{~nm}(1.55 \mathrm{eV})$ to both induce the Stark shift and to generate white-light supercontinuum in a sapphire crystal in order to measure the reflectivity of the strongly coupled exciton-polariton structure at normal incidence (Figure 1a). The sample consists of GaAs QWs placed at each of the three antinodes of two $\mathrm{Ga}_{0.8} \mathrm{Al}_{0.2} \mathrm{As} / \mathrm{AlAs}$ distributed Bragg reflectors (DBR) which form the cavity with 16 and 20 layers for the front and back reflector, respectively. The Bragg layer thickness was tapered to allow tuning of exciton and cavity resonances with respect to each other by moving the beam on the sample. The sample was held at $10 \mathrm{~K}$ in a cryostat in all experiments. In Figure $1 \mathrm{~b}$, the normalized reflectivity is shown at various detunings, vertically offset for clarity. The central spectrum, where the polariton signatures at $1.6 \mathrm{eV}$ and $1.608 \mathrm{eV}$ are equally large, defines optimum coupling. All experiments were performed in this setting. The differential reflectance $\Delta R / R$ was measured taking into account the background and photoluminescence signal similar to [9]. Pump and probe were cross-linearly polarized to allow additional stray light reduction.
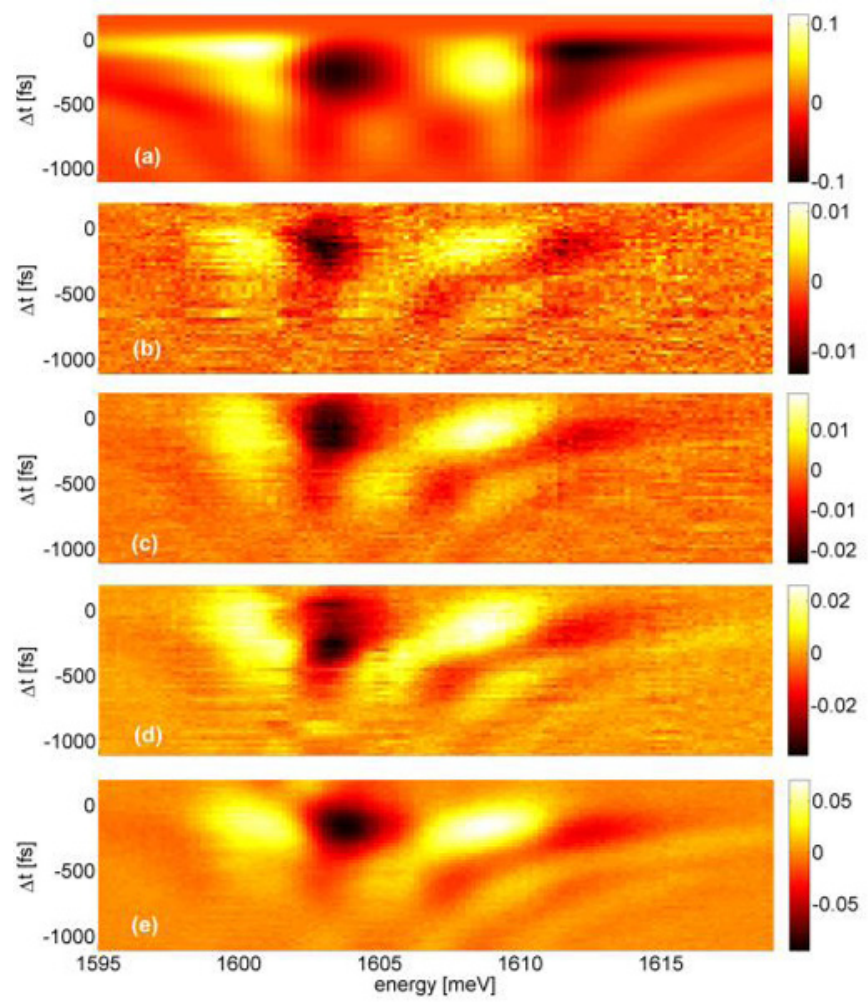

Fig. 2. Calculated differential reflection spectra as a function of pump-probe delay for a pump fluence of $0.2 \mathrm{mJcm}^{-2}$ (a). Measured differential spectra for various pump-probe time-delays for a pump fluence of $0.2 \mathrm{mJcm}^{-2}$ (b), $0.6 \mathrm{mJcm}^{-2}$ (c), $1.2 \mathrm{mJcm}^{-2}$ (d), and $2 \mathrm{mJcm}^{-2}(\mathrm{e})$.

In Figure 2, $\Delta R / R$ is plotted in false colours as a function of energy and time. In the experimental data (b-e), coherent oscillations [10] of the polarization are observed for negative pump-probe delays. They are used to determine time zero similar to Ref. [9]. Around $t=0$, two distinct dispersive features are observed in $\Delta R / R$. The neighbouring decrease and increase of reflectivity results from a shift of the respective polariton peak. We determine a shift of $\Delta E=0.5 \mathrm{meV}$ for the highest pump fluence (e). Results from our calculation (a) based on diagonalizing the system Hamiltonian, 
including both the vacuum interaction and the non-resonant pump field and accounting for coherent effects [10], display good agreement with our experiment.

The shift scales linearly with fluence, as evident from Figure 3 , where $\Delta E$ is plotted as a function of pump fluence. Inset (a) shows absolute reflection curves for unshifted (red line) and shifted (blue line) polariton doublet, and (b) shows $\Delta R / R$ at maximum fluence and signal amplitude, i.e., at $t=-100$ fs.

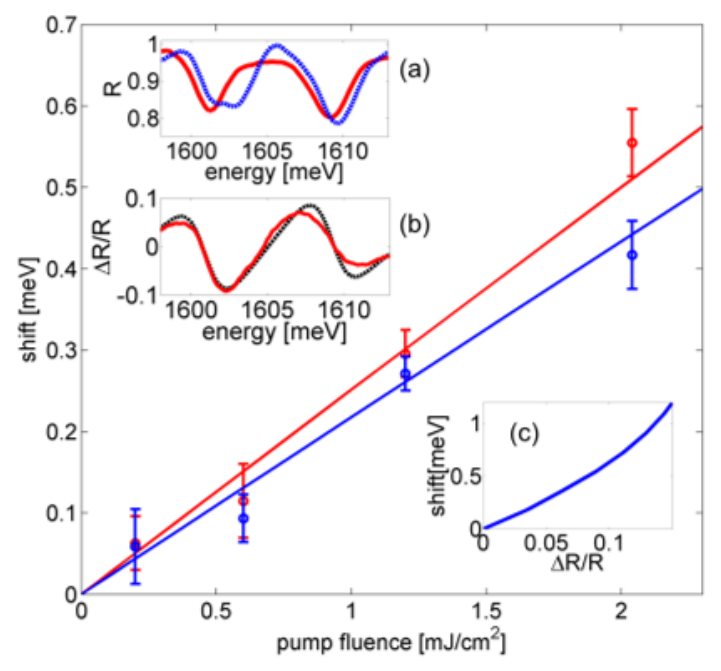

Fig. 3. Blue shift vs. pump fluence with calculated dependence for lower (red) and upper (blue) polariton. (a) Reflectivity of initial (red) and shifted (blue) polariton doublet. (b) $\Delta R / R$ at $t=0$ and a fluence of $2 \mathrm{mJcm}^{-2}$, both measured (solid red line) and calculated (black dashed line). (c) Maximum amplitude of $\Delta R / R$ vs. corresponding shift.

In conclusion, we have provided experimental evidence for an ultrafast and noninvasive shift of a polariton doublet in a strongly coupled semiconductor microcavity exploiting the dynamic Stark effect. Using high-intensity, red-detuned pump pulses, the doublet was blue-shifted by up to $0.5 \mathrm{meV}$ without altering its energy structure. Our scheme may provide a useful tool for BEC interference experiments, offering ultrafast control over potentials in a polariton condensate.

1. R. J. Thompson, G. Rempe, and H. J. Kimble, Phys. Rev. Lett. 68, 1132 (1992)

2. M. Brune, F. Schmidt-Kaler, A. Maali, J. Dreyer, E. Hagley, J. M. Raimond, and S. Haroche, Phys. Rev. Lett. 76, 1800 (1996)

3. C. Weisbuch, M. Nishioka, A. Ishikawa, and Y. Arakawa, Phys. Rev. Lett. 69, 3314 (1992)

4. H. Deng, G. Weihs, C. Santori, J. Bloch, and Y. Yamamoto, Science 298, 199 (2002)

5. J. Kasprzak, M. Richard, S. Kundermann, A. Baas, P. Jeambrun, J. M. J. Keeling, F. M. Marchetti, M. H. Szymanska, R. André, J. L. Staehli, V. Savona, P. B. Littlewood, B. Deveaud and Le Si Dang, Nature 443, 409 (2006)

6. E. Wertz, L. Ferrier, D. D. Solnyshkov, R. Johne, D. Sanvitto, A. Lemaître, I. Sagnes, R. Grousson, A. V. Kavokin, P. Senellart, G. Malpuech and J. Bloch, Nature Phys. 6, 860 (2010)

7. D. Sanvitto, S. Pigeon, A. Amo, D. Ballarini, M. De Giorgi, I. Carusotto, R. Hivet, F. Pisanello, V. G. Sala, P. S. S. Guimaraes, R. Houdré, E. Giacobino, C. Ciuti, A. Bramati and G. Gigli, Nature Photon. 5, 610 (2011)

8. Y. Shin, M. Saba, T. A. Pasquini, W. Ketterle, D. E. Pritchard, and A. E. Leanhardt, Phys. Rev. Lett. 92, 050405 (2004)

9. C. Lange, N. S. Köster, S. Chatterjee, H. Sigg, D. Chrastina, G. Isella, H. von Känel, M. Schäfer, M. Kira, and S. W. Koch, Phys. Rev. B 79, 201306(R) (2009)

10. M. Lindberg and S. W. Koch, J. Opt. Soc. Am. B 5, 139 (1988) 